\title{
Primary Intracranial Germinoma: Case Report and Review of Literature
}

\author{
K Messoudi ${ }^{1}$, K Oualla ${ }^{1}$, N Acharfi ${ }^{1}$, 0 Zouiten$^{1}$, I Ouafki ${ }^{1}$, L Amaadour ${ }^{1}$, Z Benbrahim ${ }^{1}$, FZ \\ M'rabet, S Arifi ${ }^{1}$, Y Dkhissi ${ }^{2}$, A Amarti ${ }^{3}$, M Maaroufi ${ }^{3}$ and N Mellas ${ }^{1}$ \\ ${ }^{1}$ Department of Medical Oncology, Hassan II University, Morocco \\ ${ }^{2}$ Department of radiology, Hassan II University, Morocco \\ ${ }^{3}$ Laboratory of Pathology, Morocco
}

*Corresponding author: K Messoudi, Department of Medical Oncology-Hassan II University Hospital-Fez, Morocco.

To Cite This Article: K Messoudi. Primary Intracranial Germinoma: Case Report and Review of Literature. Am J Biomed Sci \& Res. 2019 - 5(4). AJBSR.MS.ID.000923. DOI: 10.34297/AJBSR.2019.05.000923.

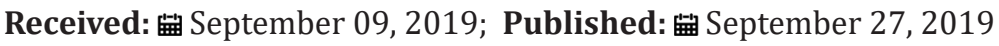

\begin{abstract}
Primary intracranial germinomas (PIGs) are an extremely rare entity with usual location in the pineal and suprasellar regions. Adult cases are rare since they are commonly diagnosed in the second decade of life. Diagnosis is based on specific imaging features in addition to tumor markers, cerebrospinal fluid cytology and stereotactic biopsy. We report a rare case of pineal germinoma diagnosed in adult patient at department by cerebrospinal fluid cytology. The therapeutic strategy was based on neoadjuvant chemotherapy followed by radiotherapy. The patient received chemotherapy based on BEP regimen (bleomycine-etoposide-cisplatin) with a clear clinical benefit from the first cycle of chemotherapy by complete resolution of clinical symptoms. The evolution was marked after the third cycle of chemotherapy by severe febrile pancetopenia leading to septic complications and the death of patient.
\end{abstract}

The aim of this work is to describe the rarity of this case, clinical radiological and histological features, in addition to therapeutic management and discuss them in comparison with literature.

Keywords: Brain; Germinoma; Pineal Region; Chemotherapy; Radiotherapy

\section{Introduction}

Primary intracranial germinomas (PIGs) are rare malignant brain tumors that represent approximately $0.2 \%$ to $1.7 \%$ of all primary intracranial tumors (1). The usual anatomical locations of these tumors are pineal, parapineal and suprasellar regions. The less common sites include intrasellar region, fourth ventricle, occipital region and cauda equine.

Case Representation
Total surgical resection of cerebral germ cell tumors GCTs is limited by the deep anatomical location of these tumors. Therefore, treatment is mainly based on chemotherapy and craniospinal irradiation. Advances in diagnostic imaging, surgical and radiotherapy and the adjunction of chemotherapy have significantly improved the outcome of patients with these tumors.
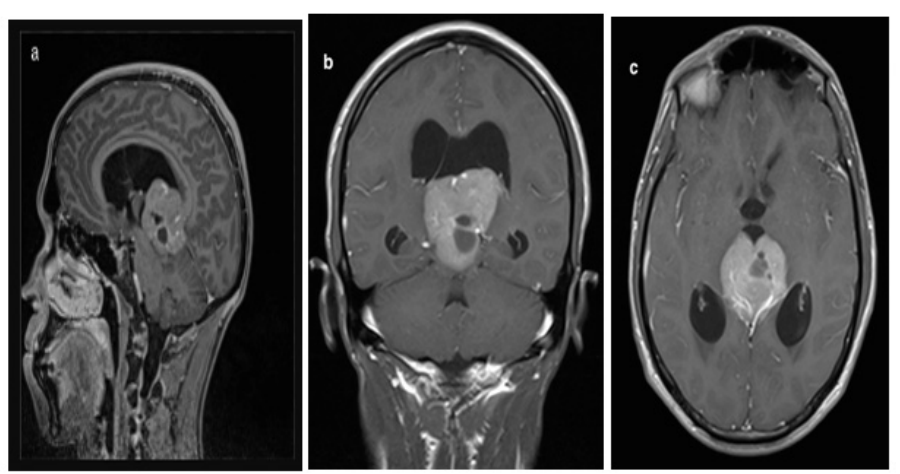

Figure 1a, b, c: MRI T1-weighted postcontrast views: tumorous expansion of solid-cystic nature in the pineal region (arrows), penetrating the 3rd ventricle with compressed aqueduct of Silvius and 3-ventricular hydrocephalus. 
We report the case of a 23-years-old male with an unremarkable past medical history who was admitted in the department of medical oncology at Hassan II University Hospital for headache rebellious to symptomatic treatment; associated to diplopia. Neurological examination did not reveal any neurological deficit. Cerebral Computed tomography (CT) scan showed an expansive process of the pineal area measuring $35.5 * 44 * 52.5 \mathrm{~mm}$ with double component containing calcifications. A subsequent gadolinium-enhanced magnetic resonance imaging (MRI) revealed a bulky expansive process of the pineal area, isointense T2 and T1 compared to gray sub- stance (Figure 1a-1c). The lesion measured $4 * 4 * 4.6 \mathrm{~cm}$ and caused considerable masse effect and biventricular hydrocephalus.

These neuroradiological features evoked a germinoma of the pineal area. Tumor markers were assessed at the moment of diagnosis and showed a normal value of alpha-fetoprotein (AFP) and a slightly high level of beta-human chorionic gonadotropin (b-HCG) at $7.87 \mathrm{IU} / \mathrm{L}(\mathrm{n}=0-5 \mathrm{IU} / \mathrm{L})$. A lumbar puncture was performed, and the cytology of the cerebrospinal fluid confirmed the diagnosis of the germinoma (Figure 2a). Patient underwent a medullar MRI and thoracic scan that did not show metastases (Figure 2b).
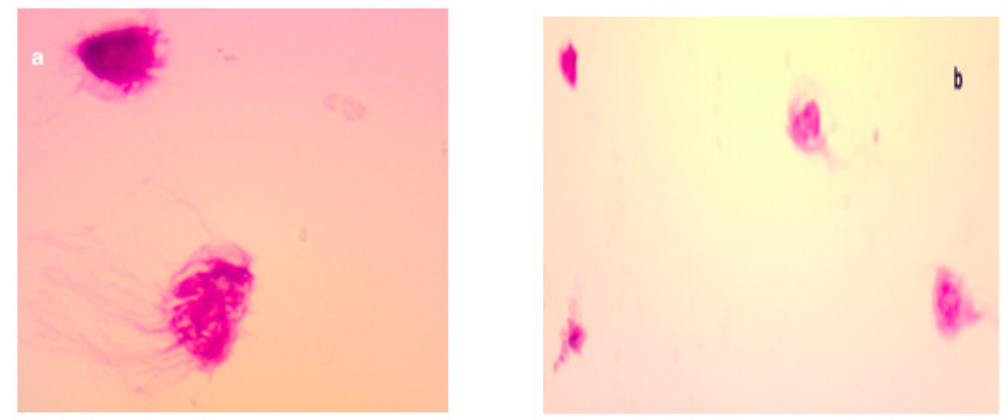

Figure 2a: The tumor cells have irregular nuclei and nuclei $\left({ }^{*} 100\right)$.

Figure 2b: Presence of large tumor cells mixed with small lymphocytes ( $\left.{ }^{*} 400\right)$.

The case was discussed in a multidisciplinary meeting and the decision was to start neoadjuvant chemotherapy followed by radiotherapy. After normal blood exams and normal cardiac and respiratory functions, the patient received the chemotherapy regimen BEP based on: Etoposide $100 \mathrm{mg} / \mathrm{m}^{2}$ IV (5 days), Cisplatin $20 \mathrm{mg} / \mathrm{m}^{2}$ IV (5days) and Bleomycin 30 units IV weekly on Days 1,8, and 15.

The evolution was marked by a clear clinical benefit from the first cycle of chemotherapy with complete resolution of diplopia and headaches. After the third cycle, the patient was admitted for worsening of performance status, with febrile pancytopenia without any identified infection at microbiological exams. He received antiobitherapy based on piperacillin/tazobactam with transfusion, but the evolution was unfortunately marked by septic complications with rapid worsening of vital functions leading to the death of patient.

\section{Discussion}

Germ cell tumors (GCTs) arise almost exclusively from midline axis. The intracranial location interests mainly pineal gland and the suprasellar area with pineal tumors that occur nearly twice as often as suprasellar GCTs. Intracranial GCTs can also arise in other intracranial areas such as the cerebral hemisphere, basal ganglia, thalamus, and cerebellum [1,2]. These tumors are usually pure germinomas and they are located in both pineal and supra-sellar sites in 5 to $10 \%$ of cases $[3,4]$. Tumor seeding or multiple tumor nodules along the lateral and third ventricles are observed in about $10 \%$ of patients. Intracranial GCT is diagnosed mainly in young patients especially during the second decade of life, with a median age at diagnosis of 10 to 12 years; and a male predominance (sex ratio be- tween 2:1 and 3:1), especially for tumors of the pineal region $[3,4]$.

Many molecular alterations were found, by analyzing molecular pathogenesis of intracranial GCTs such as isochromosome $12 \mathrm{p}$ $[5,6]$ and gain-of-function mutations of KIT $[7,8]$. Next generation sequencing (NGS) was used in an international collaborative study, to analyze 62 intracranial GCTs and revealed several alterations that may therapeutically targeted. Overall, $53 \%$ of tumors harbored somatic mutations in at least one of the genes involved in the KIT/ RAS or AKT/mTOR signalling pathways [9].

Clinically, the symptoms depend on the location of the tumor. Obstructive hydrocephalus is a usual consequence of pineal tumors in addition to symptoms related to high intracranial pressure such as headache, vomiting, papilledema and somnolence in 25 to $50 \%$ of cases. Other signs might be seen including ataxia and behavioral changes. Neuro-opthalmologic abnormalities such as paralysis of upward gaze and convergence are present in up to $50 \%$ of cases [10]. Delays in diagnosis are common, especially endocrinal symptoms that may occur with delays of more than 12 months and are associated with higher rate of metastatic disease [11] but generally the endocrinopathies are rarely associated with pineal tumors at diagnosis [12].

Diagnosis of intracranial GCTs can be retained based on increased level of tumor markers including secreted into the cerebrospinal fluid (CSF) and serum, as well as by the presence of histochemical markers on tumor cells. The markers including beta-hCG and AFP are measured in the CSF and serum. Additional immunohistochemical analysis may confirm the diagnosis by using placental alkaline phosphatase (PLAP) and c-Kit on tumor cells. 
Intracranial GCTs may be divided into two groups: "secreting" and "nonsecreting" tumors. Secreting tumors are commonly defined as tumors with CSF AFP > 10 microg/L and/or a CSF beta-hCG level $>50$ int. unit/L. Secreting GCTs are more more aggressive with poorer outcomes. Pure germinomas generally are negative for AFP and beta-hCG levels in both CSF and serum and only a minority of germinomas present a high level of beta-hCG levels in the CSF and/ or serum $[13,14]$.

Radiologically, magnetic resonance imaging (MRI) is the best imaging tool for more accurate diagnosis and for staging. Otherwise, the computed tomography (CT) also has high sensitivity to detect suprasellar and pineal GCTs. On MRI, intracranial GCTs present an iso-intense appearance or hypo-intense on T1 sequences and hyperintense on T2 sequences. These tumors typically show homogeneous enhancement with gadolinium or heterogeneous enhancement if cysts are present. Imaging characteristics of the histologic subtypes are similar, and MRIs cannot reliably distinguish germinomas from NGGCTs $[15,16]$. It is imperative to complete with MRI of the entire spine in order to stage adequately the intracranial GCTs, because 10 to $15 \%$ of cases may have leptomeningeal spread $[3,12]$

Measurement of AFP and beta-hCG in the CSF is more sensitive than serum levels in detecting abnormalities. However, discordance between serum and CSF tumor marker results has been observed, and both of them should be measured especially if no contraindication to lumbar puncture in addition to its usefulness for cytology. In case of contraindication to lumbar puncture, then tumor markers from ventricular CSF may be considered for diagnosis [14].

Histologically, the pathological proof is needed to establish a definitive diagnosis of an intracranial GCT and to ascertain the histologic subtype. A tissue sample should be obtained especially in case of normal CSF and serum AFP and beta-hCG to distinguish between pure germinoma or mature teratoma and other benign and malignant lesions, including pineal primitive neuroectodermal tumor (PNET), ependymoma (pineal location), craniopharyngioma, Langerham cell histiocytosis (suprasellar location), low-grade glioma, hamartoma, or metastatic disease from extra-cranial tumors unless surgery cannot be performed safely [17].

Patients with positive CSF cytology are considered as metastatic intracranial GCTs despite the normality of the spine MRI and must receive craniospinal irradiation (CSI). For patients with a suspected intracranial GCT, surgery for the confirmation of histologic diagnosis is strongly recommended in the absence of contraindications. Surgery is also indicated in case of neuro-surgical complication such as obstructive hydrocephalus from a pineal mass or for acute visual deterioration from a suprasellar mass [18]. When there is discordance between the tissue histological diagnosis and the CSF and/or serum markers, the treatment should be based upon the result that is associated with the most malignant histology and worst prognosis.
The gross total resection of localized germinomas is generally not recommended because of post-surgical complications and because pure germinomas are very radiosensitive [17]. Most contemporary series have reported exciting results with radiation therapy alone with long-term progression free survival (PFS) rates superior to $90 \%$ for patients with pure germinomas $[19,20]$. The historical schema of radiotherapy was 36 Gy craniospinal irradiation (CSI) and a boost to the primary tumor for a total of 50 to $54 \mathrm{~Gy}$. Recent studies showed that whole-brain or whole-ventricle irradiation in patients with localized germinomas resulted in a spinal failure rate of less than 10 percent [17,21-23]. Therefore, whole-ventricle RT with additional boost to the tumor therefore became the new standard in the treatment of localized germinoma [24]. Several clinical researches are working on reducing both the dose and volume of radiation, without compromising the excellent survival rate to overlook the neurocognitive and endocrine commplications of radiotherapy. Germinomas are also highly chemo sensitive. Therefore, recent studies have evaluated the addition of chemotherapy to reduced dose of RT in order to minimize late complications [24,25].

Platinum-based chemotherapy regimens have showna high level of activity against extracranial GCTs in children. As in adults with advanced GCTs, the most widely used combinations are bleomycin, etoposide, and either cisplatin (BEP) or carboplatin [26]

The excellent results obtained among children and adults with extracranial GCTs, have led to the exploration of neoadjuvant chemotherapy in patients with localized intracranial germinomas, followed by a reduced dose and volume of RT, in an effort to minimize toxicity. Several series have shown excellent tumor response to chemotherapy suggesting that neoadjuvant chemotherapy allows for the reduction of both the dose and volume of RT in patients with localized germinomas without compromising PFS [26,27]. While it is clear that the addition of chemotherapy may allow reduction of radiation volume, larger studies with longer follow-up have shown that further elimination of whole-ventricle RT increases the tumor recurrence rate $[24,25,28]$.

In a series of 60 patients with localized germinomas, neoadjuvant chemotherapy followed by $40 \mathrm{~Gy}$ focal RT to the tumors resulted in eight-year EFS of 83\%. Eight of 10 recurrences occurred outside the RT field, in the periventricular area [25].

In the SIOP CNS GCT 96 prospective nonrandomized study, 190 patients with localized germinomas received either chemotherapy plus 40 Gy focal RT or 24 Gy CSI with a 16 Gy tumor boost without chemotherapy $[28,29]$. The five-year EFS for patients receiving chemotherapy and focal RT was less than for those receiving RT to a larger field without chemotherapy (88 versus 94 percent). In the patients who received chemotherapy plus focal RT, six of seven recurrences (86 percent) were ventricular, either alone or in combination with local tumor recurrence. In the patients who received CSI, all four relapses were at the original tumor site. 
A preliminary report of a phase II study from the Japanese CNS GCT Study Group described outcomes in 123 patients with localized germinomas, most of whom were treated with chemotherapy followed by focal RT [24]. The recurrence rate was higher in patients who received chemotherapy plus focal RT compared to those who received chemotherapy plus whole ventricle RT (28 versus 6 percent). As discussed above, the current standard of care for radiation alone (without neoadjuvant chemotherapy) in localized germinomas is 21 to 24 Gy to the whole ventricle and an additional boost to the tumor for a total dose of 40 to $45 \mathrm{~Gy}$. Despite the promise of neoadjuvant chemotherapy, further reduction of the total RT dose below 40 Gy and/or elimination of whole-ventricle RT should only be done within the context of a prospective randomized trial

The current COG trial for localized germinomas is examining the efficacy of reducing the dose of whole ventricle irradiation (to $18 \mathrm{~Gy}$ ) and local tumor boost (to $12 \mathrm{~Gy}$ ) in patients whose tumors had complete responses to chemotherapy and compare to historical outcomes.

Although nearly all patients with localized germinomas respond to chemotherapy, treatment with chemotherapy alone has resulted in unacceptable tumor recurrence rates. In two series that included a total of 64 patients with pure germinomas, recurrent disease eventually developed in 48 and 58 percent, respectively [30,31]. The Third International CNS Germ Cell Tumor Study also confirmed that a chemotherapy-only approach led to inferior event-free survival compared to radiation-containing regimens [32].

In our case, the patient had an expansive process of the pineal area, the lesion measured $4 * 4 * 4.6 \mathrm{~cm}$ and caused considerable masse effect and biventricular hydrocephalus.

The case was discussed in a multidisciplinary meeting and the decision was to start a neoadjuvant chemotherapy followed by radiotherapy. Our patient was received three cycles of chemotherapy and the evolution was marked by a clear clinical benefit from the first cycle of chemotherapy, but the patient died after septic complications with febrile pancytopenia.

\section{Conclusion}

Primary intracranial germinomas are rare malignant brain tumors and diagnostically very challenging. Recognition of this rare form of localization of GCT by imaging is important in addition to confirmation with tumor markers, cerebrospinal fluid cytology and stereotactic biopsy if needed. Treatment is based on multimodal therapy including mainly radiotherapy and chemotherapy. Further molecular research is required in order to better understand these tumors and develop new drugs.

\section{References}

1. Kim DI, Yoon PH, Ryu YH, Jeon P, Hwang GJ (1998) MRI of germinomas arising from the basal ganglia and thalamus. Neuroradiology 40(8): $507-$ 511.

2. Tamaki N, Lin T, Shirataki K, Hosoda K, Kurata H, et al. (1990) Germ cell tumors of the thalamus and the basal ganglia. Childs Nerv Syst 6(1): 3-7.

3. Jennings MT, Gelman R, Hochberg F (1985) Intracranial germ-cell tumors: natural history and pathogenesis. J Neurosurg 63(2): 155-167.

4. Hoffman HJ, Otsubo H, Hendrick EB, Humphreys RP, Drake JM, et al. (1991) Intracranial germ-cell tumors in children. J Neurosurg 74(4): 545-551.

5. Palmer RD, Foster NA, Vowler SL, I Roberts, CM Thornton, et al. (2007) Malignant germ cell tumours of childhood: new associations of genomic imbalance. Br J Cancer 96(4): 667-676.

6. Okada Y, Nishikawa R, Matsutani M, Louis DN (2002) Hypomethylated X chromosome gain and rare isochromosome $12 p$ in diverse intracranial germ cell tumors. J Neuropathol Exp Neurol 61(6): 531-538.

7. Kamakura Y, Hasegawa M, Minamoto T, Yamashita J, Fujisawa H (2006) C-kit gene mutation: common and widely distributed in intracranial germinomas. J Neurosurg 104(3 Suppl): 173-180.

8. Sakuma Y, Sakurai S, Oguni S, Satoh M, Hironaka M, Saito K (2004) c-kit gene mutations in intracranial germinomas. Cancer Sci 95(9): 716-720.

9. Wang L, Yamaguchi S, Burstein MD, Terashima K, Chang K, et al. (2014) Novel somatic and germline mutations in intracranial germ cell tumours. Nature 511(7508): 241-245.

10. Andrew G Lee, Paul Brazis, Evelyn A Paysse, Douglas R (2017) Nordli Supranuclear disorders of gaze in children.

11. Packer RJ, Cohen BH, Cooney K (2000) Intracranial germ cell tumors. Oncologist 5: 312 .

12. Sethi RV, Marino R, Niemierko A, Tarbell NJ, Yock TI, et al. (2013) Delayed diagnosis in children with intracranial germ cell tumors. J Pediatr 163(5): 1448-1453.

13. Ogino H, Shibamoto Y, Takanaka T, Suzuki K, Ishihara S, et al. (2005) CNS germinoma with elevated serum human chorionic gonadotropin level: clinical characteristics and treatment outcome. Int J Radiat Oncol Biol Phys 62(3): 803-808.

14. Shibamoto Y, Takahashi M, Sasai K (1997) Prognosis of intracranial germinoma with syncytiotrophoblastic giant cells treated by radiation therapy. Int J Radiat Oncol Biol Phys 37(3): 505-510.

15. Liang L, Korogi Y, Sugahara T, Ikushima I, Shigematsu Y, et al. (2002) MRI of intracranial germ-cell tumours. Neuroradiology 44(5): 382-388.

16. Ogiwara H, Tsutsumi Y, Matsuoka K, Kiyotani C, Terashima K, et al. (2015) Apparent diffusion coefficient of intracranial germ cell tumors. J Neurooncol 121(3): 565-571.

17. Matsutani M, Sano K, Takakura K, Fujimaki T, Nakamura O, et al. (1997) Primary intracranial germ cell tumors: a clinical analysis of 153 histologically verified cases. J Neurosurg 86(3): 446-455.

18. Souweidane MM, Krieger MD, Weiner HL, Finlay JL (2010) Nervous system germ cell tumors: proceedings from the Second International Symposium on Central Nervous System Germ Cell Tumors. J Neurosurg Pediatr 6(2): 125-130.

19. Bamberg M, Kortmann RD, Calaminus G, Becker G, Meisner C, et al. (1999) Radiation therapy for intracranial germinoma: results of the German cooperative prospective trials MAKEI 83/86/89. J Clin Oncol 17(8): 2585-2592.

20. Jensen AW, Laack NN, Buckner JC, Schomberg PJ, Wetmore CJ, et al. (2010) Long-term follow-up of dose-adapted and reduced-field radiotherapy with or without chemotherapy for central nervous system germinoma. Int J Radiat Oncol Biol Phys 77(5): 1449-1456.

21. Haddock MG, Schild SE, Scheithauer BW, Schomberg PJ (1997) Radiation therapy for histologically confirmed primary central nervous system germinoma. Int J Radiat Oncol Biol Phys 38(5): 915-923.

22. Dattoli MJ, Newall J (1990) Radiation therapy for intracranial germinoma: the case for limited volume treatment. Int J Radiat Oncol Biol Phys 19(2): 429-433. 
23. Shikama N, Ogawa K, Tanaka S, Toita T, Nakamura K, et al. (2005) Lack of benefit of spinal irradiation in the primary treatment of intracrania germinoma: a multiinstitutional, retrospective review of 180 patients. Cancer 104(1): 126-134.

24. Matsutani M (2008) Treatment of intracranial germ cell tumors: the second phase II study of Japanese GCT Study Group. J Neurooncol 10: 420.

25. Alapetite C, Brisse H, Patte C, Raquin MA, Gaboriaud G, et al. (2010) Pattern of relapse and outcome of non-metastatic germinoma patients treated with chemotherapy and limited field radiation: the SFOP experience. Neuro Oncol 12(12): 1318-1325.

26. Allen JC, DaRosso RC, Donahue B, Nirenberg A (1994) A phase II trial of preirradiation carboplatin in newly diagnosed germinoma of the central nervous system. Cancer 74(3): 940-944.

27. Cheng S, Kilday JP, Laperriere N, Janzen L, Drake J, et al. (2016) Outcomes of children with central nervous system germinoma treated with multi-agent chemotherapy followed by reduced radiation. J Neurooncol 127(1): 173-180.

28. Calaminus G, Alapetite C, Frappaz D, Rolf-Dieter Kortmann, Maria Luisa Garre, et al. (2008) Outcome of localized and metastatic germinoma treated according to SIOP CNS GCT 96. J Neurooncol 10: 420.
29. Calaminus G, Kortmann R, Worch J, Nicholson JC, Alapetite C, et al. (2013) SIOP CNS GCT 96: final report of outcome of a prospective, multinational nonrandomized trial for children and adults with intracranial germinoma, comparing craniospinal irradiation alone with chemotherapy followed by focal primary site irradiation for patients with localized disease. Neuro Oncol 15(6): 788-796.

30. Kellie SJ, Boyce H, Dunkel IJ, Diez B, Rosenblum M, et al. (2004) Intensive cisplatin and cyclophosphamide-based chemotherapy without radiotherapy for intracranial germinomas: failure of a primary chemotherapy approach. Pediatr Blood Cancer 43(2): 126-133.

31. Balmaceda C, Heller G, Rosenblum M, Diez B, Villablanca JG, et al. (1996) Chemotherapy without irradiation-a novel approach for newly diagnosed CNS germ cell tumors: results of an international cooperative trial. The First International Central Nervous System Germ Cell Tumor Study. J Clin Oncol 14(11): 2908-2915.

32. da Silva NS, Cappellano AM, Diez B, Cavalheiro S, Gardner S, et al. (2010) Primary chemotherapy for intracranial germ cell tumors: results of the third international CNS germ cell tumor study. Pediatr Blood Cancer 54(3): 377-383. 